\title{
ESTUDO DA AFLATOXINA NO AMENDOIM, DA COLHEITA À INDUSTRIALIZAÇÃO, NA REGIÃO DE SANTA ADELIA, S.P.*
}

HOMERO FONSECA **

\section{RESUMO}

Neste trabalho foi investigada, na Região de Santa Adélia, SP, a incidência de aflatoxina no amendoim (Arachis hypogaea L.) em três estágios de seu ciclo de industrialização: a) ao ser entregue à fábrica, Epocas I e II ; b) durante o armazenamento, Epocas III e IV e c) após a extração do óleo (farelo), Épocas V e VI. Em cada estágio foram feitas duas coletas de 10 amostras carda, num total de 40 amostras de amendoim e 20 de farelo.

Dos resultados pôde-se concluir que: 1) das 60 amostras apenas uma não continha aflatoxina; 2) o nível, em termos de aflatoxina $\mathrm{B}_{1}$, foi elevado, com $55 \%$ das amostras na categoria de toxidez "Alta" e 31,6\% na categoria "Muito Alta"; 3) os níveis foram mais elevados nos Estágios 1 e 2 (amendoim) decrescendo no Estágio 3 (farelo) ; 4) cinco amostras estavam excessivamente tóxicas, com mais de $10,00 \mathrm{ppm} ; 5$ ) o amendoim chega à fábrica já com elevado teor de aflatoxina, não tendo sido possível detectar, nesta região, influência do armazenamento.

\section{INTRODUÇÃO}

Em 1960 uma doença, aparentemente nova, começou a grassar nas granjas inglesas, matando em poucos meses mais de 100.000 peruzinhos. As aves morriam em curto prazo com perda de apetite, fraqueza das asas, das pernas e lesões necróticas no fígado. STEVENS et alii (1960) foram os primeiros a descrevê-la logo seguidos por outros pesquisadores dentre os quais WANNOP (1960) e WILEY (1960). Ela foi batizada de "doença " $X$ " dos perus" (BLOUNT, 1961) tendo sido logo verificado que rações contendo farelo de amendoim eram o fator comum em todos os surtos (ASPLIN e CARNAGHAN, 1961) ao mesmo tempo que entre nós AMARAL (1961) relacionava a morte de suinos com alimentos

* Entregue para publicação em 10/12/1976

* Departamento de Tecnologia Rural 
contendo torta de amendoim. Isolado o princípio tóxico verificou-se que ele era composto de quatro substâncias às quais foi dado o nome genérico de aflatoxina pois verificou-se que eram metabólitos tóxicos produzidos pelo fungo Aspergillus flavus Link (SARGEANT et alii, 1961) que se desenvolvia sobre o amendoim após a colheita. Esses metabólitos foram denominados de aflatoxinas $B_{1}, B_{2}, G_{1}$ e $G_{2}$ devido às suas fluorescências azuis e esverdeadas. Hoje sabe-se que o fungo produz vários outros metabólitos.

Nos anos que se seguiram verificou-se que o problema era quase universal pois em todos os países, ex regiões tropicais e sub-tropicais, a ocorrência de aflatoxina era relatada: na Nigéria (McDONALD e HARKNESS, 1963), na África do Sul (SELLSCHOP et alii, 1965) em Gambia (CROWTHER, 1966), na Malásia (HAN KUO e GIM SAY, 1966) e no Senegal (TOURY, 1966).

A incidência da aflatoxina em nosso país, mais precisamente em nosso Estado, tem sido grande, conforme o demonstram os trabalhos de MENEZES et alii (1966), TANGO et alii (1967) e FONSECA (1968). Em virtude da importância deste problema resolvemos levar mais adiante nossos estudos procurando conhecer qual estágio da agro-indústria do amendoim, dentro das condições prevalecentes em nosso Estado, em que houve maior incidência da aflatoxina: se com o lavrador - desde a colheita até o momento da entrega do amendoim à fábrica - ou se nas mãos da indústria - durante o armazenamento da matéria-prima e até o sub-produto estocado, que pode ser torta ou farelo.

A região escolhida para esta investigação foi a Araraquarense, pelo fato de, em pesquisa anterior (FONSECA, 1968), ter apresentado níveis de aflatoxina mais elevados que em outras regiões, como também por ter sido constatada uma incidência mais elevada das aflatoxinas do grupo das $\mathbf{G}$.

\section{MATERIAL E MÉTODOS}

O material utilizado neste trabalho constou de amostras de amendoim em casca e farelo de amendoim da safra das águas de 1967/68 coletadas na fábrica de óleo localizada no município de Santa Adélia. A fábrica e as práticas agrícolas da região em que está situada têm as seguintes características predominantes: a fábrica tem capacidade de processamento de 24 toneladas diárias de amendoim em casca. O armazenamento da matéria-prima é feito em tulhas de onde é conduzida por esteiras rolantes para os descorticadores. A matéria-prima fica bem protegida durante todo o armazenamento.

Nesta região o amendoim é arrancado com o auxílio de sulcadores e disposto horizontalmente na leira onde é deixado por 4 ou 5 dias para 
secagem, (independentemente das condições do tempo) após o que é batido e ensacado.

As amostragens foram feitas nas seguintes datas:

\begin{tabular}{|c|c|c|c|c|}
\hline poca I: & $14 / 2 / 1968$ & (amend & em & sca) \\
\hline Época II: & $6 / 3 / 1968$ & $"$ & $"$ & $"$ \\
\hline Época III: & $27 / 3 / 1968$ & $"$ & $"$ & $"$ \\
\hline poca IV: & $10 / 4 / 1968$ & $"$ & $"$ & $"$ \\
\hline V: & $10 / 4 / 1968$ & (farelo & e & no \\
\hline IV: & $21 / 5 / 1968$ & $(")$ & & $"$ \\
\hline
\end{tabular}

Em cada Epoca foram coletadas 10 amostras e numeradas, pela ordem de recolhimento, de 1 a 10, dando um total geral de 60 amostras.

Nas Épocas I e II as 10 amostras foram retiradas de 10 caminhões diferentes, à medida que iam chegando à fábrica. De cada um deles foi retirada uma amostra média, representando no mínimo 10 sacos. Estas duas Epocas compõem o Estágio 1.

Nas épocas III e IV as amostras foram tomadas da matéria-prima estocada nos armazens tendo-se o cuidado de retirar parcelas do maior número de sacos possível. Estas duas Épocas integram o Estágio 2.

Nas Épocas V e VI foi usado o mesmo critério anterior, porém foram colhidas amostras de farelo de amendoim. Estas duas Epocas perfazem o Estágio 3.

Cada amostra de amendoim em casca foi constituída de aproximadamente $5 \mathrm{~kg}$ e as de farelo, entre 2 e $3 \mathrm{~kg}$. As amostras de amendoim foram descascadas e as amêndoas trituradas em máquina de moer carne, bem homogeneizadas e peneiradas em peneiras de crivo de 1680 micra (10 "mesh") e a seguir desengorduradas em extrator de Soxhlet, por 8 horas. Os farelos foram triturados em moinho de discos e peneirados.

A toxina foi extraída de acordo com o método de LEE (1965) e dosadas pelo método de COOMES e FEUELL (1965). As amostras foram analisadas em triplicata e os resultados expressam a média obtida nas três determinações.

Para enquadramento das amostras, quanto à sua toxidez, foi utilizada a tabela do TROPICAL PRODUCTS INSTITUTE (1962) e que está baseada nos efeitos de testes biológicos com marrequinhos de um dia (QUADRO I). 
QUADRO I - Relação entre concentração de aflatoxina $B_{1}$ e toxidez.

NÍVEL DE AFLATOXINA $B_{1}$

Abaixo de $0,05 \mathrm{ppm}$

Entre 0,05 e $0,25 \mathrm{ppm}$

Entre 0,25 e $1,00 \mathrm{ppm}$

Acima de $1,00 \mathrm{ppm}$
Baixa ou negativa

Média

Alta

Muito Alta

Para a análise da variância dos resultados, em termos de aflatoxina $\mathbf{B}_{1}$, foram tomados em cada amostra os valores encontrados para aflatoxina $B_{1}$ somados à metade dos valores de $G_{1}$, visto que esta tem cerca de $50 \%$ da toxidez de $B_{1}$ (CARNAGHAN et alii, 1963). Estas variáveis (y) foram transformadas em $\log _{10}(y+1)$ de acordo com SNEDECOR (1956) e STEEL e TORRIE (1960) pois os valores encontrados eram de magnitudes muito diferentes e incluiam também valores iguais a zero.

Foi efetuado também um estudo da evolução do teor de aflatoxina nas amostras, em função do tempo, por meio de regressão. A soma dos QUADRADOS (SQ), para tempo, foi desdobrada num componente linear, num componente de $2 .^{\circ}$ grau e em desvio de regressão. Paralelamente foram estimadas as constantes de uma equação de regressão do tipo:

$\log _{10}(\mathrm{y}+1)=\mathrm{a}+\mathrm{bx}+\mathrm{cx}^{2}$ (SNEDECOR, 1956)

onđe $\mathrm{x}=\mathrm{n} .^{\circ}$ de dias a partir do início das coletas.

\section{RESULTADOS E DISCUSSÃO}

Os resultados obtidos nas análises das aflatoxinas $B_{1}$ e $G_{1}$ encontram-se nos QUADROS II e III e os das análises estatísticas nos QUADROS IV, V e VI.

Pelos resultados pôde-se observar que apenas uma amostra estava isenta de aflatoxina e que outras 6 , representando cerca de $10 \%$ do total, apresentaram níveis baixos. A grande maioria das amostras $(86,6 \%)$ enquadrou-se nas categorias de toxidez "Alta" (55\%) e "Muito Alta" $(31,6 \%)$ QUADRO IV. Deve-se salientar o fato de que cinco amostras continham mais de 10,00 ppm de afaltoxina, teor excessivamente elevado.

O nível de aflatoxina cresceu do Estágio 1, média de 1,27 ppm, para o Estágio 2, média de 1,71 ppm, decrescendo no Estágio 3, média de 0,60 ppm, QUADRO III. A média geral foi elevada, alcançando 1,74 ppm. 
QUADRO II - Teor das aflatoxinas $B_{1}$ e $G_{1}$ amostras de amendoim e farelo de amendoim nas diversas Epocas (expresso em ppm).

\begin{tabular}{|c|c|c|c|c|c|c|c|c|c|c|c|c|}
\hline \multirow{2}{*}{ Amostra } & \multicolumn{2}{|c|}{ Epoca I } & \multicolumn{2}{|c|}{ Epoca II } & \multicolumn{2}{|c|}{ Epoca III } & \multicolumn{2}{|c|}{ Epoca IV } & \multicolumn{2}{|c|}{ Epoca V } & \multicolumn{2}{|c|}{ Epoca VI } \\
\hline & $\mathrm{B}_{1}$ & $G_{1}$ & $\mathrm{~B}_{1}$ & $\mathrm{G}_{1}$ & $\mathrm{~B}_{1}$ & $\mathrm{G}_{1}$ & $\mathrm{~B}_{1}$ & $\mathrm{G}_{1}$ & $\mathrm{~B}_{1}$ & $\mathrm{G}_{1}$ & $\mathrm{~B}_{1}$ & $G_{1}$ \\
\hline 1 & 0,30 & 0,12 & 0,02 & 0,0 & 0,30 & 0,12 & 1,75 & 0,66 & 0,75 & 0,12 & 0,30 & 0,12 \\
\hline 2 & 3,75 & 2,81 & 0,08 & 0,0 & 0,30 & 0,01 & 0,30 & 0,12 & 0,75 & 0,12 & 0,30 & 0,12 \\
\hline 3 & 1,75 & 0,66 & 0,02 & 0,01 & 1,75 & 1,41 & 0,02 & 0,01 & 0,75 & 0,12 & 0,30 & 0,12 \\
\hline 4 & 1,75 & 0,28 & 0,30 & 0,01 & 1,75 & 0,01 & 3,75 & 1,41 & 0,30 & 0,12 & 0,30 & 0,12 \\
\hline 5 & 15,00 & 0,12 & 0,0 & 0,0 & 0,75 & 0,66 & 0,30 & 0,28 & 0,75 & 0,28 & 0,30 & 0,12 \\
\hline 6 & 0,02 & 0,01 & 0,30 & 0,12 & 0,30 & 0,12 & 0,0 & 0,01 & 0,75 & 0,12 & 0,30 & 0,12 \\
\hline 7 & 0,30 & 0,01 & 7,50 & 2,81 & 0,30 & 0,0 & 3,75 & 1,41 & 0,75 & 0,28 & 0,30 & 0,12 \\
\hline 8 & 0,75 & 0,12 & 1,75 & 1,41 & 0,30 & 0,12 & 15,00 & 1,41 & 0,30 & 0,12 & 0,30 & 0,12 \\
\hline 9 & 0,75 & 0,12 & 0,30 & 0,28 & 1,75 & 0,01 & 7,50 & 0,28 & 0,75 & 0,12 & 0,30 & 0,12 \\
\hline 10 & 0,02 & 0,01 & 15,00 & 0,0 & 0,75 & 0,01 & 15,00 & 0,66 & 0,75 & 0,12 & 0,30 & 0,12 \\
\hline
\end{tabular}

QUADRO III - Médias parciais e geral da aflatoxina $\left(B_{1}+1 / 2 G_{1}\right)$ por Épocas e por ESTÁGIOS (expressas em ppm).

\begin{tabular}{|c|c|c|c|c|c|c|}
\hline \multicolumn{6}{|c|}{ EPOCA } & \multirow{2}{*}{$\begin{array}{l}\text { Média } \\
\text { Geral }\end{array}$} \\
\hline I & II & III & IV & V & VI & \\
\hline 1.38 & 1,16 & 0,91 & 2,84 & 0,80 & 0,42 & 1,14 \\
\hline \multicolumn{2}{|c|}{$\begin{array}{c}\text { ESTÁGIO } 1 \\
1,27\end{array}$} & \multicolumn{2}{|c|}{$\begin{array}{c}\text { ESTÁGIO } 2 \\
1,71\end{array}$} & \multicolumn{2}{|c|}{$\begin{array}{c}\text { ESTÁGIO } 3 \\
0,60\end{array}$} & \\
\hline
\end{tabular}


QUADRO IV - Distribuição das amostras por níveis de aflatoxina $\left(B_{1}+1 / 2 B_{1}\right)$ e respectivas categorias de toxidez, em números absolutos (n) e percentagens

\begin{tabular}{|c|c|c|c|}
\hline NÎVEIS (ppm) & $n$ & $\%$ & CATEGORIA DE TOXIDEZ \\
\hline $0,0-0,05$ & 7 & 11,67 & Baixa ou Negativa \\
\hline $0,05-0,25$ & 1 & 1,67 & Média \\
\hline $0,25-1,00$ & 33 & 55,00 & Alta \\
\hline $1,00-2,50$ & 8 & 13,33 & \\
\hline $2,50-5,00$ & 2 & 3,33 & Muito Alta \\
\hline $5,00-10,00$ & 4 & 6,67 & \\
\hline Acima de 10,00 & 5 & 8,33 & \\
\hline TOTAL & 50 & 100,00 & - \\
\hline
\end{tabular}

QUADRO V - Valores de F obtidos na análise da variância.

\begin{tabular}{l|c|l}
\hline Tratamento & Graus de Liberdade & $\mathrm{F}$ \\
\hline Epocas & $(5)$ & $2,31 * *$ \\
E. Estágios & 2 & $2,95 * *$ \\
D. Estágio 1 & 1 & 0,09 \\
D. Estágio 2 & 1 & $4,97 * *$ \\
D. Estágio 3 & 1 & 0,57 \\
\hline
\end{tabular}

Significância ao nível de $1 \%$ de probabilidade.

QUADRO VI - Valores de F referentes à aplicação de regressão no estudo do efeito de Epocas.

\begin{tabular}{lcc}
\hline Epocas & $(5)$ & $2,31 *$ \\
Linear & 1 & 2,65 \\
Quadrático & 1 & 2,10 \\
Desvio Regressão & 3 & 2,27 \\
Resídıo & & - \\
\hline
\end{tabular}

Significância ao nível de $5 \%$ de probabilidade. 
As amostras com os teores mais elevados foram encontrados na Época IV que também registrou a maior média: $2,84 \mathrm{ppm}$.

Como pode-se observar praticamente todo amendoim já entrou na fábrica com elevados teores de aflatoxina. O teor médio mais baixo encontrado no Estágio 3 pode ser devido à degradação térmica parcial da aflatoxina durante o aquecimento e prensagem do amendoim quando este atinge temperaturas de quase $100^{\circ} \mathrm{C}$ ou atmbém proveniente de material menos tóxico. Nãofo i possível detectar a influência do armazenamento, pois os resultados foram contraditórios.

A análise estatística revela que houve diferenças significativas, ao nível de $1 \%$ de probabilidade, entre as Épocas, entre os Estágios e dentro do Estágio 2. Dentro dos outros Estágios não houve diferenças significativas.

O estudo da variação da aflatoxina, em função do tempo, empregando-se regressão, revelou que houve diferença entre as Épocas, significativas ao nível de $5 \%$ de probabilidade, porém sem tendência alguma, dentro da equação considerada, pois tanto no efeito linear como no quadrático, os valores de F não foram significativos (QUADRO VI). A curva de regressão correspondente é mostrada na FIGURA 1.

O desvio da regressão não foi significativo o que indica que a equação foi adequada.

\section{CONCLUSÕES}

Dos resultados obtidos pode-se chegar às seguintes conclusões: aflatoxina;

2) O nível, em termos de aflatoxina $B_{1}$, foi elevado, com $55 \%$ na categoria de toxidez "Alta" e 31,6\% na categoria "Muito Alta".

3) Os níveis foram mais elevados nos Estágios 1 e 2 (amendoim em casca) decrescendo no Estágio 3 (farelo).

4) Cinco amostras estavam excessivamente tóxicas, com mais de $10,00 \mathrm{ppm}$ de aflatoxina.

5) O amendoim chega à fábrica já com elevado teor de aflatoxina, não tendo sido possível detectar, nesta região, a influência do armazenamento. 


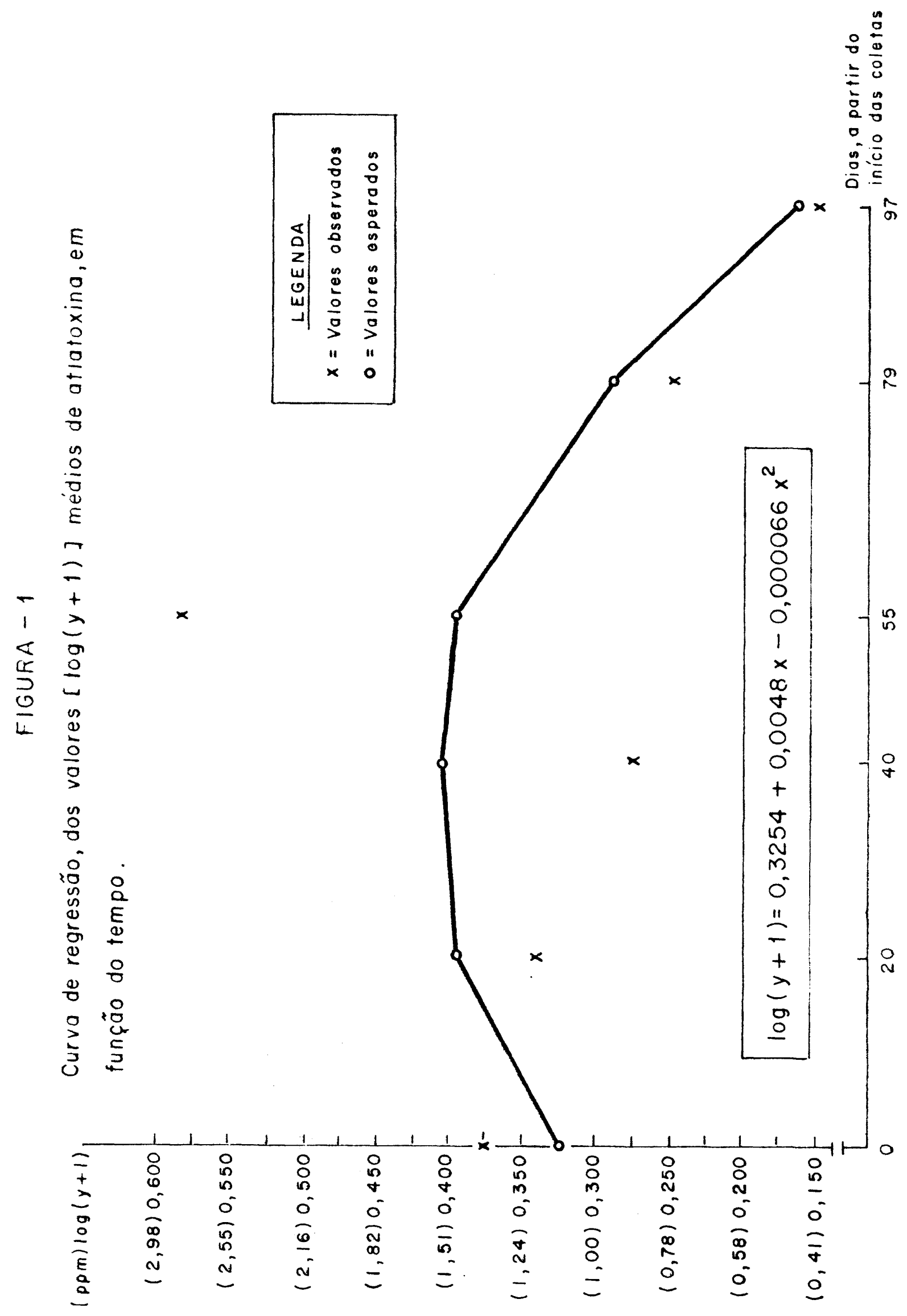




\section{SUMMARY}

\section{STUDY OF AFLATOXIN IN PEANUTS, FROM HARVEST TO INDUSTRIA- LIZATION, IN THE REGION OF SANTA ADELIA, S.P.}

In the present work the occurrence of aflatoxin in peanut (Arachis hypogaea L.) in the region of Santa Adélia, S.P., was investigated in three Stages, from harvest to industrialization: a) by the time the grower sells it to the oil mill: Epochs I and II; b) during its storage prior to milling: Epochs III and IV and c) after oil extration (peanut flour): Epochs V and VI. In each Stage two collections of 10 samples each, were made in a total of 40 samples of peanuts and 20 samples of peanut flour.

From the results the following conclusions could be drawn: 1) all samples, except one, were toxic; 2) the toxicity level, in terms of aflatoxin $\mathrm{B}_{1}$, was hight, for $55 \%$ of the samples were in the category "High" and $31.6 \%$ in the category "Very High"; 3 ) the levels of aflatoxin were higher in Stages 1 and 2 (peanuts), decreasing in Stage 3 (peanut four); 4) five samples were excessively toxic with more than $10.00 \mathrm{ppm}$; 5) the aflatoxin is already present in peanuts when the grower take it to the oil mill, but it was not possible to detect, in this region, the influence of storage.

\section{AGRADECIMENTOS}

Agradecemos à FAPESP pelo auxílio financeiro concedido e ao prof. Roland Vencovsky pela orientação recebida na execução das análises estatísticas.

\section{LITERATURA CITADA}

AMARAL, L.B.S., 1961. Torta de amendoim e morte de suinos. O Biológico, 27 (3) : 63.

ASPLIN, F.D. e R.B.A. CARNAGHAN, 1961. The toxicity of certain groundnut meals for poultry with special reference to their effect on ducklings and chickens. Vet. Rec., 73 (46) : 1215-19.

BLOUNT, W.P., 1961. Turkey "X" disease. Turkeys, $9(2): 52,55,58,61,67$.

CARNAGHAN, R.B.A., R.D. HARTLEY e J.O'KELLY, 1963. Toxicity and fluorescence properties of the aflatoxins. Nature $200: 1101$.

COOMES, T.J. e A.J. FEUELL, 1965 - Recommended procedures for the detection and estimation of aflatoxin $B_{1}$ in groundnuts and groundnut materials. Tropical Products Institute, Report G 13, Ministry of Overseas Development, Londres.

CROWTHER, P.C., 1966. Report of the Produce Chemist: $4^{\text {th }}$ November $1965-4^{\text {th }}$ May 1966. Yundum Experimental Station, Department of Agriculture, The Gambia. 15 p. xerografadas. 
FONSECA, H., 1968. "Contribuição ao estudo da ocorrência de aflatoxina em tortas, farelos e farinhas de amendoim (Arachis hypogaea L.) do Estado de São Paulo". ESALQ/ USP, Piracicaba, 65 p. Tese de Doutoramento.

HAN KUO, L. e Y. GIM SAY, 1966. The occurrence of aflatoxin in Malayan, imported oil cakes and groundnut kernels. The Malaysian Agric, J., 45 (3) 232-244.

LEE, W.V., 1965. Quantitative determination of aflatoxin in groundnut products. Analyst, 90 (1070) : 305-307.

McDONALD, D. e C. HARKNESS, 1963. Growth of Aspergillus flavus and production of aflatoxin in groundnuts. Part II. Trop. Sci., 5 (3) : 143-154.

MENEZES, T.J.B., J.S. TANGO, F.A.S. COELHO e C.G. TEIXEIRA, 1966. Ocorrência do Aspergillus flavus e da aflatoxina em sementes e farelo de amendoim. XVIII Reunião Anual da S.B.P.C., Blumenau, S.C.

SARGEANT, K., A. SHERIDAN, J. O’KELly e R.B.A. CARNAGHAN, 1961. Toxicity associated with certain samples of groundnuts. Nature, 192: 1096-1097.

SELLSCHOP, J.P.F., N.P.J. KRIEK e J.C.G. du PREEZ, 1965. Distribution and degree of occurrence of aflatoxin in groundnuts and groundnut products. Symp. Mycotoxins Foodstuffs, Agric. Aspects, Febr. 1965. Pretoria, South Africa 9-17.

SNEDECOR, G.W., 1956. Statistical Methods. 5. ${ }^{\mathrm{a}}$ ed. The Iowa State College Press, Ames, Ia, 534 pp.

STEEL, R.G.D. e J.H. TORRIE, 1960. Principles and Procedures of Statistics. McGraw Hill Book, Co, N. York, 481 pp.

STEVENS, A.J., C.N. SAUNDERS, J.B. SPENCE e A.G. NEWHAM, 1960. Investigation into "diseases" of turkey poults. Vet. Rec., 72 (31): 627-628.

TANGO, J.S., T.J.B. MENEZES e C.G. TEIXEIRA, 1967. Levantamento da ocorrência da aflatoxina em sementes de amendoim nas safras das águas e da seca de 1965 . XIX Reunião Anual da S.B.P.C., Rio de Janeiro, R.J.

TOURY, J., 1966. Rapport sur l'Operation Exagraf. In: "Rapport sur les recherches effectuées sur l'aflatoxine au cours de l'année 1965-1966. O.R.A.N.A., Dakar, Senegae. $12 \mathrm{p}$.

TROPICAL PRODUCTS INSTITUTE, 1962. Aflatoxin in groundnuts and groundnut products. Interpretation of physico - chemical and biological test results. T.P.I. Ministry of Overseas Development, Londres, $1 \mathrm{p}$.

WANNOP, C.C. 1960. Disease of turkey poults. Vet. Rec. 72 (33) 671-672.

WILEY, J.R., 1960. "Disease" of turkey poults. Vet. Rec., 72 (38) 786-787. 